\title{
Remdesivir in Coronavirus Disease 2019 (COVID-19) treatment: a review of evidence
}

\author{
Hui Xian Jaime Lin ${ }^{1} \cdot$ Sanda $\mathrm{Cho}^{2} \cdot$ Veeraraghavan Meyyur Aravamudan ${ }^{1} \cdot$ Hnin Yu Sanda ${ }^{3}$ Raj Palraj ${ }^{4}$. \\ James S. Molton ${ }^{5} \cdot$ Indumathi Venkatachalam $^{6}$
}

Received: 27 July 2020 / Accepted: 16 November 2020 / Published online: 2 January 2021

○) Springer-Verlag GmbH Germany, part of Springer Nature 2021

\begin{abstract}
COVID-19 is an infectious disease caused by a novel $\beta$-coronavirus, belonging to the same subgenus as the Severe Acute Respiratory Syndrome (SARS) virus. Remdesivir, an investigational broad-spectrum antiviral agent has previously demonstrated in vitro activity against Severe Acute Respiratory Syndrome Coronavirus 2 (SARS-CoV-2), and in vivo efficacy against other related coronaviruses in animal models. Its safety profile has been tested in a compassionate use setting for patients with COVID-19. The current therapeutic studies demonstrate clinical effectiveness of remdesivir in COVID-19 patients by shortening time to clinical recovery, and hospital stay. In this review, we critically analyze the current evidence of remdesivir against COVID-19 and dissect the aspects over its safety and efficacy. Based on existing data, remdesivir can be regarded as a potential therapeutic agent against COVID-19. Further large-scale, randomized placebo-controlled clinical trials are, however, awaited to validate these findings.
\end{abstract}

Keywords COVID-19 · SAR-CoV-2 $\cdot$ Remdesivir $\cdot$ Hui Xian Jaime Lin and Sanda Cho shared the first authorship

\section{Introduction}

In December 2019, the novel Coronavirus Disease 2019 (COVID-19) was first identified as a new emerging infectious disease in China. It has rapidly spread across the globe, and was declared a global health emergency by the World Health Organization (WHO) on 30th January 2020 [1]. To date, many countries are still struggling to contain the spread of this virus. As of 2 nd November 2020, there are among

Hui Xian Jaime Lin

jaimelin06@gmail.com

1 Department of Medicine, Woodlands Health Campus, Singapore, Singapore

2 Department of General Medicine, Warrington and Halton Hospitals NHS Trust, Warrington, UK

3 Department of Acute Medicine, Queens Hospital, Romford, England, UK

4 Division of Infectious Diseases, Mayo Clinic College of Medicine, Rochester, USA

5 Department of Infectious Disease, Western Health, Footscray, Australia

6 Department of Infectious Diseases, Singapore General Hospital, Singapore, Singapore
218 countries affected, 46.8 million infections and over 1.2 million deaths reported worldwide [2]. These numbers continue to rise daily.

COVID-19 is caused by a novel $\beta$-coronavirus, a ribonucleic acid (RNA) virus which belongs to the same subgenus as the Severe Acute Respiratory Syndrome (SARS) virus [3]. It has, therefore, been designated Severe Acute Respiratory Syndrome Coronavirus 2 (SARS-CoV-2) by the Coronavirus Study Group of the International Committee on Taxonomy of Viruses [3]. SARS-CoV-2 shares 79\% of the sequence homology with SARS-CoV and more distantly (50\% of sequence homology) with the Middle East Respiratory Syndrome Coronavirus (MERS-CoV) [4]. These are two Coronaviruses that had caused severe acute respiratory syndrome outbreaks in China in 2002/2003 and in Saudi Arabia in 2012, respectively [5].

The clinical course of COVID-19 ranges from asymptomatic infection or mild respiratory symptoms to severe or life-threatening pneumonia and death. With no definitive curative treatment insight, and high mortality rate in vulnerable populations, health authorities have sought to re-stratify risks, and focus on the repurposing of available drugs to develop timely and cost-effective therapeutic strategies, targeting the hospitalized and critically ill. Several 
antiviral/antimalarial agents such as remdesivir, ritonavir/ lopinavir combination, hydroxychloroquine, chloroquine; and immuno-modulating therapies such as tocilizumab, sarilumab, lenzilumab, eculizumab, ravulizumab, convalescent plasma, and interferon are currently being evaluated in randomized controlled trials (RCT) in many countries to evaluate their efficacy and safety in the treatment of COVID-19.

Remdesivir was touted as a potential candidate drug for the treatment of COVID-19. Recent studies have shown promising results and have been regarded as a 'molecule of hope' for the treatment of COVID-19 [6]. On the 22nd October 2020, remdesivir became the first United States Food and Drug Administration (FDA) approved drug for the treatment of hospitalized COVID-19 patients [7]. Our objective is to review and summarize the most current evidence of the antiviral properties of remdesivir, its safety profile and efficacy in COVID-19 patients. We will also briefly discuss other experimental treatments that are available in the literature.

\section{Remdesivir as an anti-viral agent: in vitro and in vivo studies}

Remdesivir, also named as GS-5734 is an adenosine analogue with a broad-spectrum antiviral activity against RNA viruses [8]. It is a prodrug that requires metabolism by the host cell to its active form, GS-441524, that interferes with viral RNA-dependent RNA polymerase ( $R d R p)$ enzyme causing a delay in chain termination, arresting RNA synthesis and viral replication [8].

In vitro, remdesivir has been shown to inhibit viral replication in both MERS-CoV and SARS-CoV [9]. Sheahan et al. measured intracellular genomic and subgenomic viral
RNA via quantitative reverse transcriptase polymerase chain reaction in remdesivir-treated human airway epithelial cell line [9]. A dose dependent reduction for both SARS-CoV and MERS-CoV was demonstrated, which is consistent with titer reduction [9].

Similarly, in mouse models of SARS-CoV infection, prophylactic or early administration of remdesivir lowered viral load and reduced SAR-CoV-associated pulmonary pathology of denuding bronchiolitis, perivascular inflammatory infiltrates and intra-alveolar edema, and prevented deterioration in pulmonary function [9]. Interestingly, therapeutic remdesivir treatment in SARS-CoV after virus replication and lung epithelial cell damage had peaked did not alter disease severity or mortality, despite a significant reduction in SARS-CoV lung viral titre [9].

These findings are consistent with studies on non-human primates (rhesus macaque model). A reduction in lung viral load was observed in both prophylactic and therapeutic treatment groups [10]. Respiratory rate of animals treated prophylactically with remdesivir remained normal throughout the study; however, $83 \%$ of those treated therapeutically developed increased heart rate [10]. Pulmonary pathology was absent in the prophylactic treated group [10]. Gross lung lesions were observed in $83 \%$ of the animals treated therapeutically with remdesivir, the total lung area affected was, however, significantly smaller than that compared to control animals [10].

Evidence from the above in vitro and in vivo studies of SARS-CoV and MERS-CoV (refer to Table 1 and Table 2) suggest that prophylactic treatment with remdesivir inhibit viral replication, prevent clinical disease and changes in pulmonary pathology [9-12]. The clinical benefit
Table 1 Summary of IC50/ IC90 or EC50/EC90 values determined by infectious viral titre from in vitro and animal studies, respectively

\begin{tabular}{|c|c|c|c|c|}
\hline Study & Component & Virus & Cell line & $\begin{array}{l}\text { EC50/EC90 (deter- } \\
\text { mined by infectious } \\
\text { viral titre) }\end{array}$ \\
\hline \multirow[t]{3}{*}{ Sheahan et al. (2017) [9] } & Remdesivir & MERS-CoV & Calu3 2B4 & $\mathrm{IC} 50=0.025 \mu \mathrm{M}$ \\
\hline & Remdesivir & MERS-CoV & $* \mathrm{HAE}$ & $\mathrm{IC} 50=0.074 \mu \mathrm{M}$ \\
\hline & Remdesivir & SARS-CoV & $*$ HAE & $\mathrm{IC} 50=0.069 \mu \mathrm{M}$ \\
\hline Wang et al. (2020) [11] & Remdesivir & SARS-CoV-2 & Vero E6 & $\begin{array}{l}\mathrm{EC} 50=0.77 \mu \mathrm{M} \\
\mathrm{EC} 90=1.76 \mu \mathrm{M}\end{array}$ \\
\hline \multirow[t]{5}{*}{ Pruijssers et al. (2020) [12] } & Remdesivir & SARS-CoV-2 & Calu3 2B4 & $\begin{array}{l}\text { EC50 }=0.28 \mu \mathrm{M} \\
\text { EC90 }=2.48 \mu \mathrm{M}\end{array}$ \\
\hline & Remdesivir & SARS-CoV-2 & Vero E6 & $\begin{array}{l}\text { EC50 }=1.65 \mu \mathrm{M} \\
\text { EC90 }=2.40 \mu \mathrm{M}\end{array}$ \\
\hline & Remdesivir & SARS-CoV-2 & $*$ HAE & $\begin{array}{l}\text { IC50 }=0.010 \mu \mathrm{M} \\
\text { IC90 }=0.009 \mu \mathrm{M}\end{array}$ \\
\hline & GS-441524^ & SARS-CoV-2 & Vero E6 & $\begin{array}{l}\text { EC50 }=0.47 \mu \mathrm{M} \\
\text { EC90 }=0.71 \mu \mathrm{M}\end{array}$ \\
\hline & GS-441524^ & SARS-CoV-2 & Calu3 2B4 & $\begin{array}{l}\mathrm{EC} 50=0.62 \mu \mathrm{M} \\
\mathrm{EC} 90=1.34 \mu \mathrm{M}\end{array}$ \\
\hline
\end{tabular}

*HAE $=$ Primary human airway epithelial

${ }^{\wedge} \mathrm{GS}-441524$ is the main plasma metabolite of the antiviral prodrug remdesivir 
Table 2 Summary of IC50/IC90 or EC50/EC90 values determined by qRT-qPCR from in vitro and animal studies, respectively

\begin{tabular}{|c|c|c|c|c|}
\hline Study & Component & Virus & Cell line & $\begin{array}{l}\text { EC50/EC } 90 \\
\text { (determined by qRT-qPCR) }\end{array}$ \\
\hline \multirow[t]{4}{*}{ Pruijssers AJ et al. (2020) [12] } & Remdesivir & SARS- COV-2 & Vero E6 & $\begin{array}{l}\text { EC } 50=1.49 \mu \mathrm{M} \\
\text { EC } 90=3.03 \mu \mathrm{M}\end{array}$ \\
\hline & Remdesivir & SARS-COV-2 & Calu3 2B4 cells & $\begin{array}{l}\text { EC } 50=0.60 \mu \mathrm{M} \\
\text { EC } 90=1.28 \mu \mathrm{M}\end{array}$ \\
\hline & GS-441524^ & SARS- COV-2 & Vero E6 & $\begin{array}{l}\text { EC } 50=0.47 \mu \mathrm{M} \\
\text { EC } 90=0.80 \mu \mathrm{M}\end{array}$ \\
\hline & GS-441524^ & SARS-COV-2 & Calu3 2B4 cells & $\begin{array}{l}\text { EC } 50=1.09 \mu \mathrm{M} \\
\text { EC } 90=1.37 \mu \mathrm{M}\end{array}$ \\
\hline
\end{tabular}

*HAE $=$ Primary human airway epithelial

${ }^{\wedge} \mathrm{GS}-441524$ is the main plasma metabolite of the antiviral prodrug remdesivir

of therapeutic remdesivir treatment, however, is less clear. Although there is an appreciable reduction in viral load, the reduction in severity and disease progression remains unclear.

\section{Remdesivir in the clinical setting}

Remdesivir was first identified as an investigational drug to treat Ebola virus disease during the West African outbreak in 2013-2016. Although remdesivir appeared promising in preclinical studies, it did not meet efficacy and safety endpoints in a clinical trial. A randomized controlled trial (RCT) by Mulangu et al. of 681 patients with acute Ebola virus infection demonstrated remdesivir to be less effective than other monoclonal antibody therapies [13]. The study was also terminated prematurely because of high case fatality rate of $53 \%$ in the remdesivir group compared to other competitive Ebola drugs [13]. The higher fatality rate could be explained by the virology of Ebola. The Ebola virus is a RNA virus with tropism to antigen presenting cells of lymph nodes, hepatocytes and endothelium [14]. Clinical manifestation include non-specific viral syndrome followed by gastrointestinal manifestations. A proportion of patients progress to a systemic inflammatory phase with hemorrhagic complications that is associated with high mortality [14]. Furthermore, Ebola virus disease predominantly affects resource poor countries with limited supportive care, likely contributing to the higher fatality rate (Tables 1,2 ).

The first case report of remdesivir use in COVID-19 originated from the United States. This is a previously well 35-year-old gentleman with history of hypertriglyceridemia, who was admitted for monitoring and isolation [15]. He remained stable for the first 6 days of his admission. His illness progressed with persistent fevers and requirement of oxygen supplementation. Remdesivir was administered as a trial on day 7 of admission (day 11 of illness) with significant clinical improvement over the next $24 \mathrm{~h}$ [15]. Subsequent to the case report, small cohort [16] and prospective
[17] studies on compassionate use of remdesivir suggests improvement in oxygen requirement, ability to wean off ventilatory support and improved clinical outcomes (refer to Table 3). Both of these studies were limited by their small sample size, and lack of a comparator group. The study by Grein et al. was further limited by lack of viral titers to measure direct anti-viral efficacy.

In more recent months, evidence from RCTs have begun to surface. The first randomized double-blinded, placebocontrolled multicentre trial was conducted by Wang et al. in Hubei, China [18]. A total of 236 patients were enrolled in this study with a randomization of 2:1 to receive remdesivir or placebo, respectively. 8 patients in the remdesivir group and 2 patients in the control group were excluded from the per-protocol analysis as they did not commence or completed less than 5 days of treatment [18]. The primary clinical endpoint was time to clinical improvement within 28 days after randomization, based on a six-point ordinal scale [18]. Time to clinical improvement, though not significant, was shorter in the remdesivir-treated group (median 21 days vs 23 days) compared to control group [18]. 28-day mortality was similar in both groups with $14 \%$ mortality in the remdesivir and 13\% in the control group [18]. No significant differences were observed in the duration of oxygen requirement, length of hospital stay, days from randomisation to discharge, and days from randomisation to death in both groups [18]. SARS-CoV-2 RNA viral load were similar in both remdesivir and control groups from onset of symptoms to start of study treatment [18]. Over time, viral load decreased similarly in both groups [18]. This study remains inconclusive, as study recruitment was halted before target enrollment was achieved, due to declining incidence of COVID-19 in China, resulting in poor statistical power.

Another randomized, open-labeled phase 3 multi-center trial (SIMPLE-Severe trial) was conducted in multiple countries (United States, Italy, Spain, Germany, Hong Kong, Singapore, South Korea, and Taiwan) to evaluate the safety and efficacy of different dosing regimens of remdesivir (5 days 


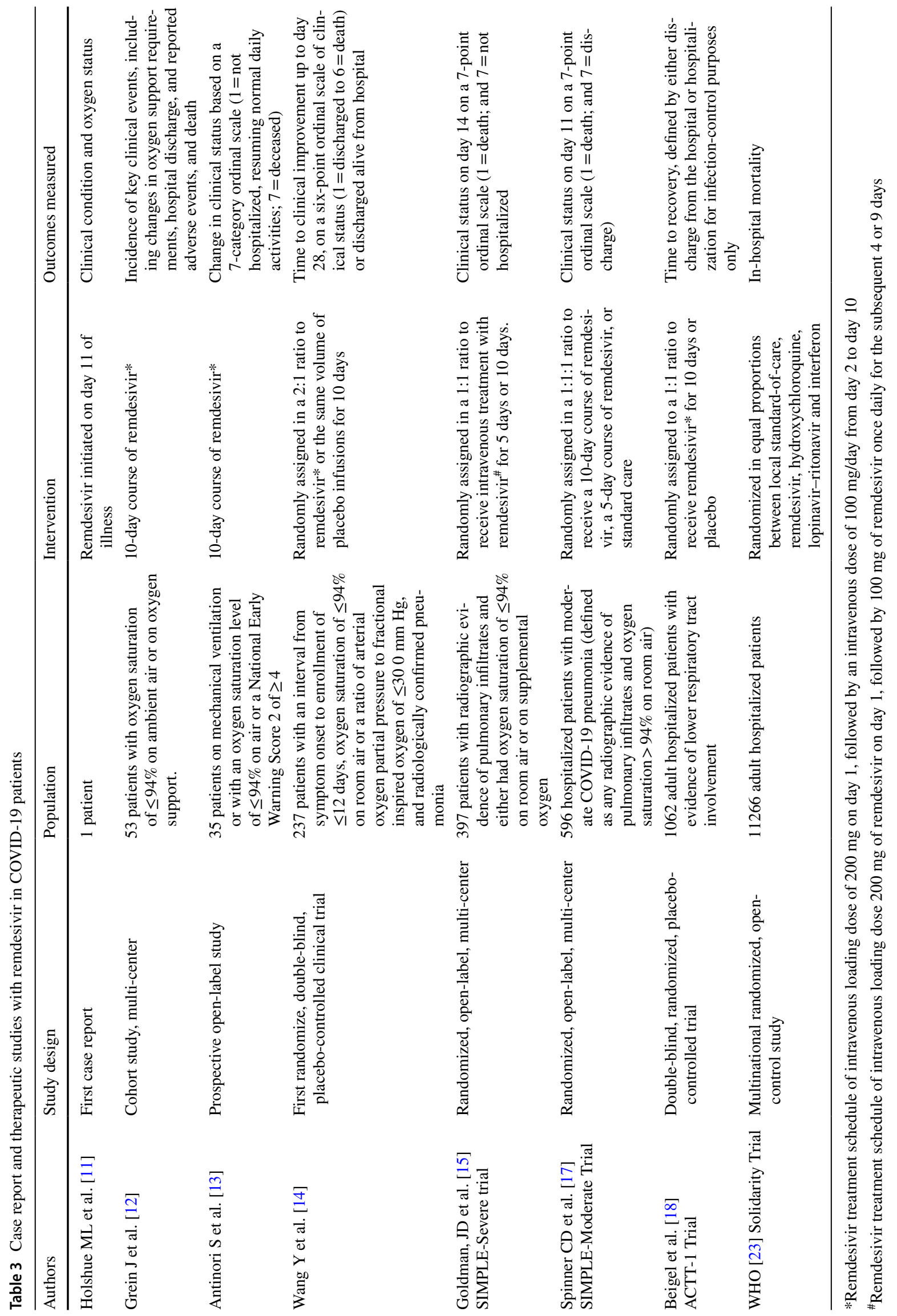




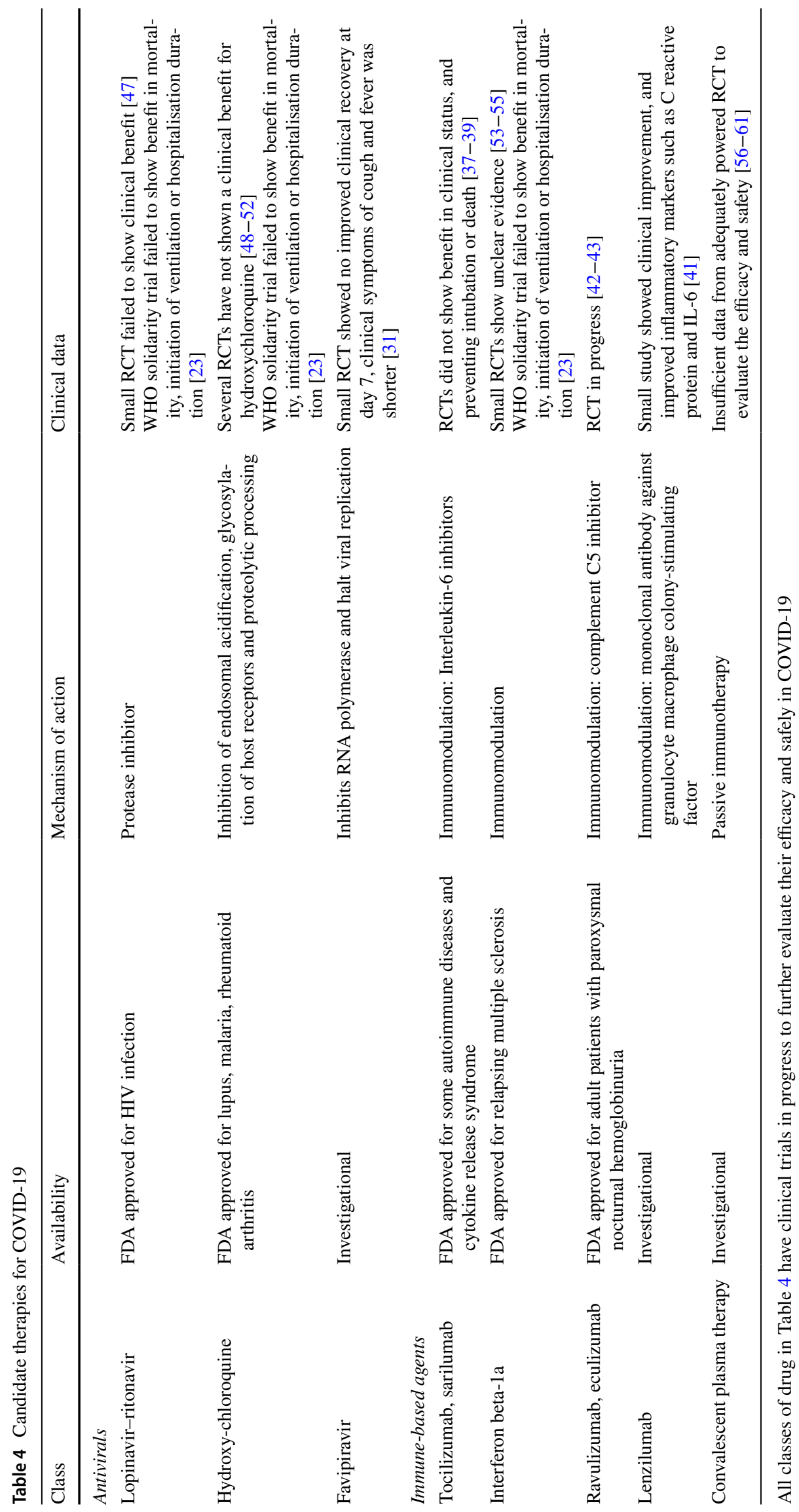


vs 10 days) in patients with severe COVID-19 [19]. 397 patients were randomized in a 1:1 ratio to receive intravenous remdesivir for either 5 or 10 days [19]. The primary efficacy endpoint was clinical status assessed on day 14 on a 7-point ordinal scale [19]. At day 14, 64\% of patients who received a 5-day course of remdesivir showed clinical improvement, as compared with 54\% of patients who received a 10-day course [19]. After adjusting for baseline clinical status, there was no difference in clinical improvement at day 14 between the groups [19]. Among patients discharged on or before day 14 , the median duration of hospitalization was similar in both the groups ( 7 days for the 5-day group and 8 days for the 10-day group) [19]. There was also no significant difference demonstrated in the number of hospital discharges and mortality, although numerically there were more discharges in the 5-day treatment group $(60 \%$ vs $52 \%$ ), and lower mortality ( $8 \%$ vs $11 \%$ ) [19]. Post hoc analysis did not demonstrate any improved outcomes with remdesivir treatment beyond 5 days among patients who were receiving non-invasive ventilation, any supplemental oxygen, or breathing ambient air [19]. In multivariate analysis, duration of clinical improvement was shorter in patients that were younger than 65 years old, of a black or white race, did not require supplemental oxygen or only required lowflow oxygen, were not on a biologic treatment and recruitment was outside Italy [19]. Efficacy of remdesivir cannot be determined in this study as it lacks a placebo control group. Although numerically, there is a trend towards better outcomes in the 5-day remdesivir-treated group, the authors have acknowledged several possible reasons. The 10-day remdesivir treatment group included a significantly higher proportion of patients with more severe COVID-19 disease, requiring invasive mechanical ventilation and high-flow oxygen [19]. Furthermore, there is a higher percentage of men in the 10-day remdesivir group [19]. Males with COVID-19 have been shown to have worse outcomes [20]. An expansion phase of the study was recently added and will include 180 trial sites worldwide and aims to enroll a further 5600 patients, including those on mechanical ventilation.

A second SIMPLE trial (SIMPLE-Moderate) was conducted to assess the safety and efficacy of standard care vs 5-day and 10-day intravenous remdesivir treatment in patients with moderate COVID-19 infection [21]. 596 patients were randomized in a 1:1:1 ratio to receive a 10-day course of remdesivir, a 5-day course of remdesivir, or standard care [21]. The primary end point was clinical status on day 11 on a 7-point ordinal scale [21]. On day 11, patients in the 5-day remdesivir group had significantly higher odds of a better clinical status distribution than those receiving standard care (odds ratio, $1.65 ; 95 \%$ CI $1.09-2.48 ; \mathrm{P}=0.02$ ) [21]. The clinical status distribution on day 11 between the 10-day remdesivir and standard care groups was not significantly different $(p=0.18)[21]$.
The National Institute of Allergy and Infectious Diseases funded ACTT-1 trial conducted a large international study of 1062 patients from 60 trial sites and 13 subsites in the United States (45 sites), Denmark (8), the United Kingdom (5), Greece (4), Germany (3), Korea (2), Mexico (2), Spain (2), Japan (1), and Singapore (1) [22]. Patients were randomized in a 1:1 ratio to receive remdesivir for 10 days or placebo [22]. The primary outcome was the time to recovery assessed on the eight-category ordinal scale [22]. Results showed that remdesivir treatment shortened recovery time by 5 days (median 10 days vs 15 days, $\mathrm{P}<0.001$ ) [22]. Furthermore, at day 15 , the odds of recovery according to the ordinal scale score were higher in the remdesivir group compared to the placebo group (odds ratio for improvement, 1.50 ; 95\% CI $1.2-1.9 ; p=0.001$ ) [22]. Although numerically, mortality rate by day 15 was lower in the remdesivir group, this was not significant $(6.7 \%$ vs $11.9 \%$, hazard ratio for death, 0.55 ; 95\% CI 0.36-0.83) [22].

Subgroup analysis showed remdesivir was most beneficial in patients receiving supplemental oxygen, but not in those receiving high-flow oxygen, non-invasive or mechanical ventilation [22]. Furthermore, benefit of remdesivir was only seen in patients with $\leq 10$ days of symptoms, and not seen in patients with $>10$ days of symptoms [22].

More recently, WHO Solidarity Trial Consortium released their trial results. This is a large multinational randomized, open-control study involving over 11,000 hospitalized patients with COVID-19 from 405 hospitals across 30 countries [23]. Remdesivir was one of four available active treatment options (remdesivir, lopinavir/ritonavir, hydroxychloroquine, and interferon beta-1a). The primary outcome was to assess effects on in-hospital mortality in all COVID-19 patients, and secondary outcomes were initiation of ventilation and hospitalization duration [23]. All four interventions failed to show benefit in mortality, initiation of ventilation or hospitalisation duration [23]. One limitation of this study is the lack of data on the duration of symptoms prior to the initiation of remdesivir. Since remdesivir is likely to be beneficial in the early viral replication phase rather than the inflammatory phase, it is possible that remdesivir was administered in the later stage of COVID-19 disease that is characterized by dysregulated host immune response. These findings, therefore, should not be interpreted that remdesivir has no role in the treatment of COVID-19.

While remdesivir has been associated with higher mortality in Ebola viral disease, this has not been observed in COVID-19, possibly due to early diagnosis and treatment initiation. Given the mechanism of action of remdesivir as an antiviral that halts viral replication, these trial findings support the use of remdesivir in the early active viral replication phase in COVID-19 PCR-positive patients. If patients progress from the viral replication phase to the inflammatory phase of infection, such as patients with ARDS requiring 
mechanical ventilation, remdesivir is not effective and antiinflammatory drugs may be beneficial. Choice of therapeutics, therefore, may depend on the disease phase, where remdesivir may have a significant role in the early viral replication phase to reduce disease progression.

\section{Safety profile of remdesivir in COVID-19}

Being an investigational drug, safety data on remdesivir are limited. Initial safety profile of remdesivir was evaluated in phase 1 clinical trial for the treatment of Ebola virus disease [25]. In this study, remdesivir demonstrated a linear pharmacokinetics within the dose range of $3 \mathrm{mg}$ and $225 \mathrm{mg}$, with an intracellular half-life of more than $35 \mathrm{~h}$ [25]. This dose range was well tolerated with no evidence of hepatic or renal toxicity [25]. Multiple doses of remdesivir, however, resulted in reversible hepatocellular enzyme elevations [25].

More recent studies from COVID-19 disease provided further insight into the safety of remdesivir. Observational study from the compassionate use of remdesivir in the treatment of severe COVID-19 showed that majority (60\%) of patients reported adverse events (AE), with $23 \%$ being serious adverse events (SAEs) [16]. All SAEs, however occurred in patients requiring invasive ventilation [16]. These SAEs include multi-organ failure, septic shock, acute kidney injury, and hypotension [16]. Other commonly reported AEs include liver derangement, diarrhea, rash, renal impairment, and hypotension, which also occurred more frequently in patients requiring invasive ventilation [16]. 8\% of patients discontinued treatment with remdesivir prematurely, as its use was attributed to one for the following: worsening of preexisting renal failure, multi-organ failure, elevated hepatic enzymes, and maculopapular rash [16]. These AEs described in this study are also commonly experienced in patients with severe COVID-19 disease. Given the lack of a control group, conclusive evidence on the safety of remdesivir cannot be made.

In the most recent and largest ACTT-1 trial, results showed no evidence of significant harm with remdesivir treatment. SAE occurred in $24.6 \%$ of patients in the remdesivir group, and $31.6 \%$ of patients in the placebo group [22]. No deaths were found to be associated with the use of remdesivir treatment [22]. The most common SAE in the remdesivir group compared to the placebo group were: (i) respiratory failure (7.3\% vs $12.8 \%$ ); (ii) hypoxia or respiratory distress (1.9\% vs $2.9 \%$ ); (iii) acute kidney injury or a reduction in estimated glomerular filtration rate (eGFR) (2.6\% vs $3.3 \%$ ); (iv) septic shock (1.5\% vs $2.9 \%$ ); and (v) cardiac arrest (1.9\% vs $1.4 \%)$ [18]. Non-serious adverse events (AEs) occurred in $51.9 \%$ of patients in the remdesivir group and 57.2\% in the placebo group [22]. The most common non-serious $\mathrm{AE}$ in the remdesivir group compared to the placebo group were (i) anemia or decreased hemoglobin
(16.5\% vs $21.7 \%$ ); (ii) acute kidney injury, a reduction in eGFR or creatinine clearance, or a rise in blood creatinine ( $16.0 \% \%$ vs $20.3 \%$ ); (iii) fever ( $7.1 \%$ vs $6.2 \%$ ), (iv) hyperglycemia or a raised blood glucose level (13.7\% vs $11.8 \%)$; and (v) elevated aminotransferase levels (6.0\% vs $10.7 \%)$ [22].

Clinical trials evaluating different dosing regimens did not demonstrate any difference in AEs among groups. Overall, $70 \%$ of patients in the 5-day remdesivir group and 74\% of patients in the 10-day group experienced AEs [21]. SAE occurred in $21 \%$ in the 5-day group and $35 \%$ in the 10-day group [21]. SAEs were, however, significantly increased in the 10-day group after adjusting for baseline clinical status [21]. Consistent with other studies, majority of the SAEs occurred in patients receiving mechanical ventilation or non-invasive ventilation or high-flow oxygen [21]. The most common SAEs were acute respiratory failure (9\% 10 days vs $5 \% 5$ days) and respiratory failure (5\% 10 days vs $2 \%$ 5 days) [21].

Overall, data from clinical trials demonstrated no significant harm with remdesivir. The AEs described are similar between all studies and may suggest that these common AEs could be a result of COVID-19 severity rather than remdesivir treatment.

\section{Current therapeutic pipelines for COVID-19 infection}

One other investigational drug is also currently being evaluated for its use in COVID-19. Favipiravir is another RNA polymerase inhibitor that hinders viral replication. Its efficacy and safety profile were mostly obtained from preclinical data with influenza and Ebola virus disease [26-30]. Clinical evidence for the use of favipiravir in COVID-19 is limited. A prospective, multicentre RCT of 240 COVID19 patients was randomized in a 1:1 ratio to receive either favipiravir or arbidol [31]. Favipiravir did not significantly improve the clinical recovery rate at day 7 [31]. No difference was observed in the requirement for supplemental oxygen or non-invasive mechanical ventilation [31]. Duration of febrile illness and cough, however, was significantly shorter in the favipiravir group [31]. To better evaluate the efficacy of favipiravir in the treatment of COVID-19, RCTs with a placebo control group will need to be conducted.

The anti-cytokines and immune-modulatory agents are also being evaluated for their use in severe COVID-19 disease. Pro-inflammatory cytokines, such as interleukin (IL)-2, IL-6, IL-7, IL10, granulocyte colony-stimulating factor, interferon- $\gamma$ inducible protein 10 , monocyte chemoattractant protein 1 , macrophage inflammatory protein $1-\alpha$, and tumour necrosis factor- $\alpha$ have been implicated in the underlying pathophysiology of a dysregulated host hyperinflammatory response in severe COVID-19 disease 
[32-34]. There are ongoing clinical trials evaluating the treatment options for this dysregulated host hyperinflammation targeting the inflammatory cascade. Earlier case studies from China suggest that IL-6 may be the predominant mediator of this dysregulated immune response [35]. Peak IL-6 level is associated with severity of pulmonary complications [36]; hence, monoclonal antibodies against IL-6 could reduce inflammatory response and improve clinical outcomes.

Tocilizumab, an IL-6 receptor antagonist, has been used in a small case series of 21 patients with severe COVID19 with promising evidence [37]. Clinical improvement in respiratory function was evidenced by the reduction in oxygen requirement, and interval reduction in lung opacity on CT imaging [37]. A significant reduction in the percentage of lymphocytes and $\mathrm{C}$ reactive protein levels was also observed after tocilizumab treatment [37]. No SAE or $\mathrm{AE}$ was reported in this study [37]. In more recent RCTs, tocilizumab did not show benefit in clinical status [38], or in the prevention of intubation or death in moderately ill-hospitalized patients with Covid-19 [39].

Other immune-modulatory agents that are being investigated for the management of patients with dysregulated host hyperinflammatory response include humanized monoclonal antibody against circulating granulocyte macrophage colony-stimulating factor [40, 41] (lenzilumab) and humanized monoclonal antibodies that inhibit the late stage of complement cascade (ravulizumab [42], and eculizumab [43]).

Convalescent plasma therapy, though limited in experience and evidence, is regarded as one possible treatment of COVID-19. It is increasingly being used to urgently counter the COVID-19-associated mortality [44]. The hypothesis is that convalescent plasma of SARS-CoV patients carries antibodies against coronavirus, which may lead to phagocytosis or direct neutralization of the virus. In a study during the SARS pandemic in 2003, 80 SARS patients were given convalescent plasma [45]. $41 \%$ of patients who were treated with convalescent plasma in the first 14 days of illness were discharged by day 22 [45]. The mortality rates, however, were no different in the group who received treatment before or after 14 days of illness [45]. It is difficult to make any conclusion from this study due to several limitations. These included a non-randomized study with no placebo group for comparison, variability of antibody dosing, and lack of long-term follow-up for risk of infusion-related infections.

It has been suggested, however, that empiric usage of convalescent plasma may be detrimental in some patients as "antibody-dependent enhancement (ADE)" may lead to a more severe infection later [44]. The risk of ADE is thought to occur in a patient with pre-existing antibodies. These antibodies may cross-react, and enhance infection against another virus, or a subtype of the same virus [44].

\section{Conclusion}

In depth understanding of the emerging data related to COVID-19 is crucial to curb this pandemic. At present, remdesivir remains an investigational drug for the treatment of COVID-19. Although it is associated with shorter hospital length of stay, and a more rapid clinical convalescence, no mortality benefit has been demonstrated. These results do not provide clear evidence on the efficacy and safety of remdesivir against COVID-19. It has been suggested that remdesivir is unlikely to achieve adequate concentration in lung tissues through intravenous infusion alone because of its low tissue distribution and poor lung penetration [46]. A proposed combination of pulmonary and intravenous administration of remdesivir has been suggested for a more effective strategy for the treatment of COVID-19 [46].

With the lack of available effective treatment options to date, it is reasonable to trial remdesivir treatment in patients with severe COVID-19 disease. Larger, multi-center RCTs with a placebo control group, however, is required to confirm the efficacy and safety of remdesivir before it can be considered as a 'standard' anti-viral treatment for COVID-19.

To further clarify the role of remdesivir across the clinical spectrum of COVID-19, studies comparing different treatment strategies and/or administration routes including combination of antiviral therapy with immune-modulatory agents particularly in severe COVID-19 disease are warranted.

\section{Compliance with ethical standards}

Conflict of interest All authors declare no conflict of interest nor affiliations with or financial involvement with any commercial organisations.

\section{References}

1. Statement on the second meeting of the International Health Regulations (2005) Emergency Committee regarding the outbreak of novel coronavirus (2019-nCoV) [Internet]. [cited 2020 Jul 20]. Available from: https://www.who.int/news-room/detai 1/30-01-2020-statement-on-the-second-meeting-of-the-inter national-health-regulations-(2005)-emergency-committee-regar ding-the-outbreak-of-novel-coronavirus-(2019-ncov).

2. Coronavirus Update (Live): Cases and Deaths from COVID-19 Virus Pandemic - Worldometer [Internet]. [cited 2020 Jul 20]. Available from: https://www.worldometers.info/coronavirus/.

3. Gorbalenya AE, Baker SC, Baric RS, et al. Severe acute respiratory syndrome-related coronavirus: The species and its viruses-a statement of the Coronavirus Study Group. bioRxiv 2020 .

4. Lu R, Zhao X, Li J, et al. Genomic characterisation and epidemiology of 2019 novel coronavirus: implications for virus origins and receptor binding. Lancet. 2020;395(10224):565-74. https://doi. org/10.1016/s0140-6736(20)30251-8. 
5. Zhu N, Zhang D, Wang W, et al. A Novel Coronavirus from Patients with Pneumonia in China, 2019. N Engl J Med. 2020;382(8):727-33. https://doi.org/10.1056/nejmoa2001017.

6. Saha A, Sharma AR, Bhattacharya, et al. Probable Molecular mechanism of remdesivir for the treatment of COVID-19: need to know more. Arch Med research. 2020;S0188-4409(20):30699.

7. FDA Approves First Treatment for COVID-19. [cited 2020 Oct 22] Available from: https://www.fda.gov/news-events/press-annou ncements/fda-approves-first-treatment-covid-19.

8. Agostini ML, Andres EL, Sims AC, et al. Coronavirus susceptibility to the antiviral remdesivir (GS-5734) is mediated by the viral polymerase and the proofreading exoribonuclease. MBio. 2018. https://doi.org/10.1128/mbio.00221-18.

9. Sheahan TP, Sims AC, Graham RL, et al. Broad-spectrum antiviral GS-5734 inhibits both epidemic and zoonotic coronaviruses. Sci Trans Med. 2017. https://doi.org/10.1126/scitranslmed.aal36 53.

10. de Wit E, Feldmann F, Cronin J, et al. Prophylactic and therapeutic remdesivir (GS-5734) treatment in the rhesus macaque model of MERS-CoV infection. Proc Natl Acad Sci USA. 2020;117(12):6771-6.

11. Wang M, Cao R, Zhang L, Yang X, Liu J, Xu M, et al. Remdesivir and chloroquine effectively inhibit the recently emerged novel coronavirus (2019-nCoV) in vitro. Cell Res. 2020;30:269-71.

12. Pruijssers AJ, George AS, Schäfer A, Leist SR, Gralinksi LE, Dinnon KH, et al. Remdesivir inhibits SARS-CoV-2 in human lung cells and chimeric SARS-CoV expressing the SARS-CoV-2 RNA polymerase in mice. Cell Rep. 2010;2020:107940.

13. Mulangu S, Dodd LE, Davey RT Jr, et al. A randomized, controlled trial of ebola virus disease therapeutics. N Engl J Med. 2019;381(24):2293-303.

14. Malvy D, McElroy AK, de Clerck H, Günther S, van Griensven J. Ebola virus disease. Lancet. 2019;393(10174):936-48.

15. Holshue ML, DeBolt C, Lindquist S, et al. First case of 2019 Novel Coronavirus in the United States. N Engl J Med. 2020;382(10):929-36. https://doi.org/10.1056/nejmoa2001191.

16. Grein J, Ohmagari N, Shin D, et al. Compassionate use of remdesivir for patients with severe Covid-19. N Engl J Med. 2020. https ://doi.org/10.1056/nejmoa2007016.

17. Antinori S, Cossu MV, Ridolfo AL, et al. Compassionate remdesivir treatment of severe Covid-19 pneumonia in intensive care unit (ICU) and Non-ICU patients: clinical outcome and differences in post-treatment hospitalisation status. Pharmacol Res. 2020;158:104899.

18. Wang Y, Zhang D, Du G, et al. Remdesivir in adults with severe COVID-19: a randomised, double-blind, placebo-controlled, multicentre trial [published correction appears in Lancet. 2020 May 30;395(10238):1694]. Lancet. 2020;395(10236):1569-1578.

19. Goldman JD, Lye DCB, Hui DS, et al. Remdesivir for 5 or 10 days in patients with severe Covid-19. N Engl J Med. 2020. https://doi. org/10.1056/nejmoa2015301.

20. Richardson S, Hirsch JS, Narasimhan M, et al. Presenting characteristics, comorbidities, and outcomes among 5700 patients hospitalized with COVID-19 in the New York City area. JAMA. 2020. https://doi.org/10.1001/jama.2020.6775.

21. Spinner CD, Gottlieb RL, Criner GJ, et al. Effect of remdesivir vs standard care on clinical status at 11 days in patients with moderate COVID-19: a randomized clinical trial. JAMA. 2020;324(11):1048-57.

22. Beigel JH, Tomashek KM, Dodd LE, et al. Remdesivir for the treatment of Covid-19-final report. N Engl J Med. 2020;383(19):1813-26. https://doi.org/10.1056/NEJMoa2007764.

23. WHO Solidarity Trial Consortium. Repurposed antiviral drugs for COVID-19-interim WHO Solidarity trial results. Accessed October 15, 2020.
24. Hendaus Mohamed A. Remdesivir in the treatment of coronavirus disease 2019 (COVID-19): a simplified summary. J Biomol Struct Dyn. 2020. https://doi.org/10.1080/07391102.2020.1767691.

25. World Health Organization. WHO R\&D blueprint: ad-hoc expert consultation on clinical trials for Ebola therapeutics. Published October 2018. Accessed March 20, 2020. https://www.who.int/ ebola/drc-2018/summaries-of-evidence-experimental-therapeuti cs.pdf.

26. Furuta Y, Komeno T, Nakamura T. Favipiravir (T-705), a broad spectrum inhibitor of viral RNA polymerase. Proc Jpn Acad Ser B Phys Biol Sci. 2017;93(7):449-63.

27. Mentre F, Taburet AM, Guedj J, et al. Dose regimen of favipiravir for Ebola virus disease. Lancet Infect Dis. 2015;15(2):150-1.

28. Sissoko D, Laouenan C, Folkesson E, et al; JIKI Study Group. Experimental treatment with favipiravir for Ebola virus disease (the JIKI Trial): a historically controlled, single-arm proof-ofconcept trial in Guinea [published correction appears in PLoS Med. 2016;13(4):e1002009]. PLoS Med. 2016;13(3):e1001967.

29. Shiraki K, Daikoku T. Favipiravir, an anti-influenza drug against life-threatening RNA virus infections. Pharmacol Ther. 2020;2020:107512.

30. Kumagai Y, Murakawa Y, Hasunuma T, et al. Lack of effect of favipiravir, a novel antiviral agent, on QT interval in healthy Japanese adults. Int J Clin Pharmacol Ther. 2015;53(10):866-74.

31. Chen C, Huang J, Cheng Z. et al. Favipiravir versus Arbidol for COVID-19: a randomized clinical trial. medRxiv. Preprint posted March 27, 2020.

32. Wu D, Yang XO. TH17 responses in cytokine storm of COVID19: an emerging target of JAK2 inhibitor Fedratinib. J Microbiol Immunol Infect. 2020;53(3):368-70.

33. Huang C, Wang Y, Li X, et al. Clinical features of patients infected with 2019 novel coronavirus in Wuhan, China. Lancet. 2020;395(10223):497-506.

34. Tay MZ, Poh CM, Rénia L, et al. The trinity of COVID-19: immunity, inflammation and intervention. Nat Rev Immunol. 2020. https://doi.org/10.1038/s41577-020-0311-8.

35. Zhou F, Yu T, Du R, et al. Clinical course and risk factors for mortality of adult inpatients with COVID-19 in Wuhan, China: a retrospective cohort study. Lancet. 2020;395(10229):1054-62.

36. Russell B, Moss C, George G, et al. Associations between immune-suppressive and stimulating drugs and novel COVID19-a systematic review of current evidence. Ecancermedicalscience. 2020;14:1022. https://doi.org/10.3332/ecancer.2020.1022.

37. Xu X, Han M, Li T, et al. Effective treatment of severe COVID19 patients with tocilizumab. Proc Natl Acad Sci USA. 2020;117(20):10970-5.

38. Furlow B. COVACTA trial raises questions about tocilizumab's benefit in COVID-19. Lancet Rheumatol. 2020;2(10):e592.

39. Stone JH, Frigault MJ, Serling-Boyd NJ, et al. Efficacy of tocilizumab in patients hospitalized with Covid-19. N Engl J Med. 2020. https://doi.org/10.1056/nejmoa2028836.

40. Zhou Y, Fu B, Zheng X, et al. Aberrant pathogenic GM-CSF+T cells and inflammatory CD14 + CD16 + monocytes in severe pulmonary syndrome patients of a new coronavirus. bioRxiv. 2020 . https://doi.org/10.1101/2020.02.12.945576.

41. Temesgen Z, Assi M, Vergidis P, et al. First Clinical Use of Lenzilumab to Neutralize GM-CSF in Patients with Severe COVID-19 Pneumonia. medRxiv. 2020. https://doi. org/10.1101/2020.06.08.20125369.

42. Smith K, Pace A, Ortiz S, et al. A Phase 3 open-label, randomized, controlled study to evaluate the efficacy and safety of intravenously administered ravulizumab compared with best supportive care in patients with COVID-19 severe pneumonia, acute lung injury, or acute respiratory distress syndrome: a structured summary of a study protocol for a randomised controlled trial. Trials. 2020;21(1):639. 
43. van de Veerdonk FL, Netea MG, van Deuren M, et al. Kallikreinkinin blockade in patients with COVID-19 to prevent acute respiratory distress syndrome. Elife. 2020;9:e57555.

44. Abid MA, Nunley L, Abid MB. Could Coronavirus Disease 2019 (COVID-19) render natural immunity to re-infections? A Spotlight on the Therapeutic Pipeline. Front Immunol. 2020;11:1294.

45. Cheng Y, Wong R, Soo YO, et al. Use of convalescent plasma therapy in SARS patients in Hong Kong. Eur J Clin Microbiol Infect Dis. 2005;24:44-6.

46. Sun D. Remdesivir for treatment of COVID-19: combination of pulmonary and iv administration may offer aditional benefit. AAPS J. 2020;22(4):77.

47. Cao B, Wang Y, Wen D, et al. A trial of lopinavir-ritonavir in adults hospitalized with severe Covid-19. N Engl J Med. 2020;382:1787-99.

48. Horby P, Mafham M, Linsell L, et al. Effect of hydroxychloroquine in hospitalized patients with COVID-19: preliminary results from a multi-centre, randomized, controlled trial. medRxiv. 2020;Preprint.

49. Cavalcanti AB, Zampieri FG, Rosa RG, et al. Hydroxychloroquine with or without azithromycin in mild-to-moderate COVID-19. N Engl J Med. 2020;383(21):2041-52.

50. Furtado RHM, Berwanger O, Fonseca HA, et al. Azithromycin in addition to standard of care versus standard of care alone in the treatment of patients admitted to the hospital with severe COVID19 in Brazil (COALITION II): a randomised clinical trial. Lancet. 2020;396(10256):959-67.

51. Geleris J, Sun Y, Platt J, et al. Observational study of hydroxychloroquine in hospitalized patients with COVID-19. N Engl J Med. 2020.

52. Rosenberg ES, Dufort EM, Udo T, et al. Association of treatment with hydroxychloroquine or azithromycin with in-hospital mortality in patients with COVID-19 in New York state. JAMA. 2020;323(24):2493-502.

53. Synairgen announces positive results from trial of SNG001 in hospitalised COVID-19 patients [press release]. July 20, 2020.

54. Davoudi-Monfared E, Rahmani $\mathrm{H}$, Khalili $\mathrm{H}$, et al. Efficacy and safety of interferon beta-1a in treatment of severe COVID-19: a randomized clinical trial. Antimicrob Agents Chemother. 2020.

55. Hung IF, Lung KC, Tso EY, et al. Triple combination of interferon beta- $1 \mathrm{~b}$, lopinavir-ritonavir, and ribavirin in the treatment of patients admitted to hospital with COVID-19: an open-label, randomised, Phase 2 trial. Lancet. 2020;395(10238):1695-704.

56. Agarwal A, Mukherjee A, Kumar G, et al. Convalescent plasma in the management of moderate COVID-19 in India: an openlabel parallel-arm Phase II multicentre randomized controlled trial (PLACID Trial). medRxiv. 2020; Preprint.

57. Li L, Zhang W, Hu Y, et al. Effect of convalescent plasma therapy on time to clinical improvement in patients with severe and life-threatening COVID-19: a randomized clinical trial. JAMA. 2020;324(5):460-70.

58. Liu STH, Lin HM, Baine I, et al. Convalescent plasma treatment of severe COVID-19: a propensity score-matched control study. Nat Med. 2020;26:1708-13.

59. Gharbharan A, Jordans CCE, GeurtsvanKessel C, et al. Convalescent plasma for COVID-19: a randomized clinical trial. medRxiv. 2020; Preprint.

60. Joyner MJ, Wright RS, Fairweather D, et al. Early safety indicators of COVID-19 convalescent plasma in 5,000 patients. J Clin Invest. 2020;130(9):4791-7.

61. Joyner MJ, Senefeld JW, Klassen SA, et al. Effect of convalescent plasma on mortality among hospitalized patients with COVID-19: initial three-month experience. medRxiv. 2020. 